\title{
Identifying and reducing model structure uncertainty based on analysis of parameter interaction
}

\author{
Y. Wang, J. Dietrich, F. Voss, and M. Pahlow \\ Institute of Hydrology, Water Resources Management and Environmental Engineering, Ruhr-University Bochum, 44801 \\ Bochum, Germany
}

Received: 17 January 2007 - Revised: 11 April 2007 - Accepted: 4 May 2007 - Published: 20 June 2007

\begin{abstract}
Multi-objective optimization algorithms are widely used for the calibration of conceptual hydrological models. Such algorithms yield a set of Pareto-optimal solutions, reflecting the model structure uncertainty. In this study, a multi-objective optimization strategy is suggested, which aims at reducing the model structure uncertainty by considering parameter interaction within Pareto-optimal solutions. The approach has been used to develop a nested setup of a rainfall-runoff model, which is integrated in a probabilistic meso-/macroscale flood forecasting system. The optimization strategy aided in determining the best combination of a lumped (computationally efficient in operational real time forecasting) and a semi-distributed parameterization of the hydrological model. First results are shown for two subbasins of the Mulde catchment in Germany. The different phenomena of parameter interaction were analysed in this case study to reduce the model structure uncertainties.
\end{abstract}

\section{Introduction}

Conceptual rainfall-runoff models have been widely used for hydrological modelling at the meso- and macroscale due to their significant advantages regarding parameter estimation and in computation time, when compared to physicallybased models. Conceptual models simplify the complex natural hydrological processes. In general the model parameters can not be determined experimentally and must therefore be inferred by calibration of the hydrological model using historical data. In this paper we present an efficient strategy for automatic model calibration based on pre-calibration and parameter sensitivity analysis.

In recent years several single- and multi-objective calibration algorithms have been developed (e.g. Duan et al., 1992,

Correspondence to: Y. Wang

(yan.wang-2@rub.de)
1993; Yapo et al., 1998; Vrugt et al., 2003a, b). These algorithms use objective functions to represent the hydrograph. As a result, the calibration problem transforms into an optimization problem. Single-objective optimization algorithms, such as SCE (Shuffled Complex Evolution method, Duan et al., 1992, 1993), consider only a single criterion and calculate the "best" solution corresponding to this criterion. This kind of algorithm assumes that the input data and the model structure are correct. In the optimization process only the parameter uncertainty is evaluated. In fact, calibration based on one single objective function often results in unrealistic representations of hydrographs. One reason is that the single-objective function constrains the calibration to fit certain characteristics of the system response, while neglecting the remaining aspects. Furthermore, integration of the residuals into one value underestimates the information which is present in the data (Fenicia et al., 2007). These limitations clearly stipulate the need to constrain the calibration processes with multiple objective functions, leading towards a multi-objective view of the calibration problem. Yapo et al. (1998) formulated this problem as

$\min F(\theta)=\left\{f_{1}(\theta), f_{2}(\theta), \ldots ., f_{m}(\theta)\right\}$,

where $f_{1}(\theta) \ldots . . f_{m}(\theta)$ are the $m$ non-commensurable objective functions, which reflect the model performance with respect to the parameters $\theta$ of the model. The solution of the optimization problem (Eq. 1) results not in one single solution, but rather in a set of Pareto-optimal solutions. The Pareto-optimal solutions define the minimum uncertainty in the parameters, which can improve one specific component of $F(\theta)$ without compromising others. Hence all Paretooptimal solutions are tantamount, if no objective function is preferred. Assuming a perfect model with a model structure precisely reproducing the real world, one single optimal solution must exist. However, model structural uncertainty always exists and therefore it is impossible to find the perfect solution. The Pareto-optimal solutions may be regarded as

Published by Copernicus Publications on behalf of the European Geosciences Union. 


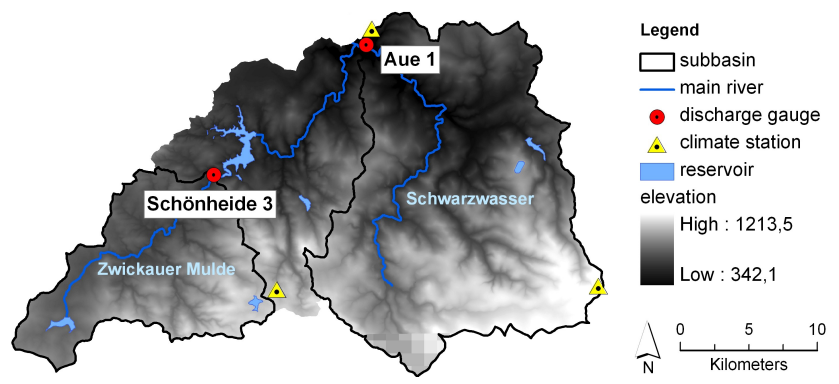

Fig. 1. Study area: sub-basins of the upper Zwickauer Mulde (Schoenheide gauge, $149 \mathrm{~km}^{2}$ ) and Schwarzwasser (Aue gauge, $363 \mathrm{~km}^{2}$ ).

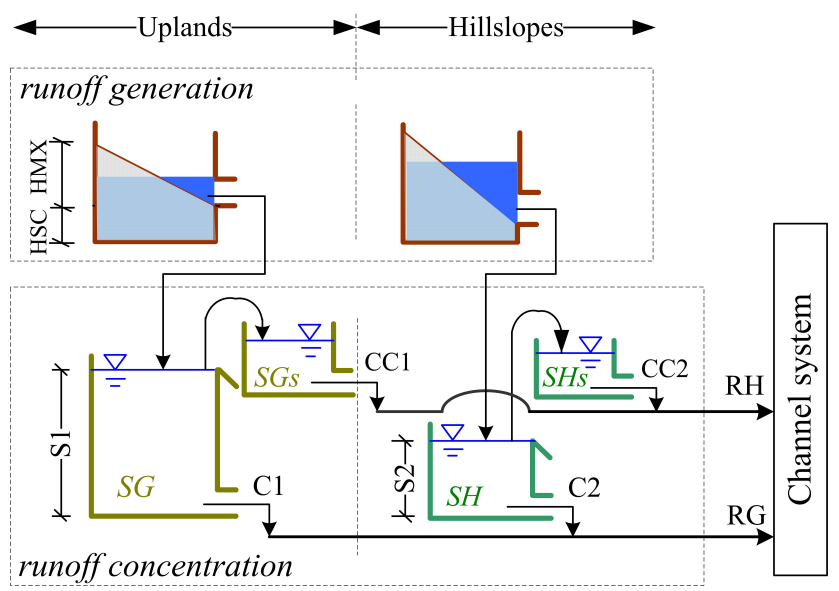

Fig. 2. ArcEGMO model structure.

failure records due to model structure uncertainty during the search for one single perfect solution.

In this study we will focus on parameter interactions, based on the analysis of Pareto-optimal solutions. As a result, a calibration strategy is proposed that improves the simulation performance of a conceptual, semi-distributed hydrological model.

\section{Data and model}

\subsection{Study area and data}

The mountainous Mulde River Basin, which lies partly in Germany and the Czech Republic, is formed by several parallel sub-basins, draining from South to North. During Westcyclonic rainfall events, which caused several extreme flood events in the past, the spatial uncertainties of the precipitation forecasts are crucial. This results in high uncertainties of flood alerts with regard to the locations of a possible inundation. For this study two upstream headwaters in the OreMountainous region have been selected (Fig. 1): the upper Zwickauer Mulde sub-basin $\left(149 \mathrm{~km}^{2}\right.$, gauge Schoenheide) (a)
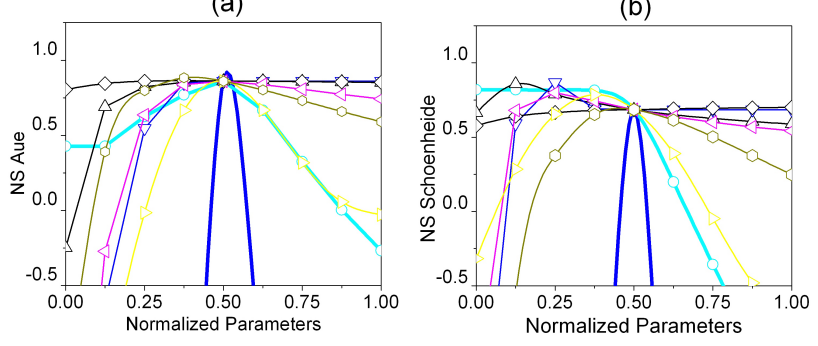

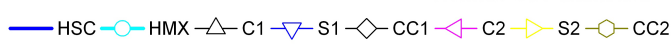

Fig. 3. Sensitivity analysis for (a) gauge Aue (Schwarzwasser) and (b) gauge Schoenheide (Zwickauer Mulde). Each parameter was changed equidistantly in its feasible space while the other parameters were fixed with the initial, manually calibrated values. The Nash Sutcliffe coefficient NS was used as performance measure. Excluding parameter interaction, the minimum storage capacity HSC of the runoff generation sub-model is the most sensitive parameter.

and the Schwarzwasser sub-basin $\left(363 \mathrm{~km}^{2}\right.$, gauge Aue). Elevation model, soil data, land use data and discharge time series were provided by local authorities. Climate time series with hourly time step were used to calibrate the model for five summer flood events (Table 1).

\subsection{Hydrological model}

The model structure of the GIS-based multi-scale hydrological modelling system ArcEGMO (Becker et al., 2002) is shown in Fig. 2. ArcEGMO consists of three sub-modules: 1) runoff generation, 2) runoff concentration and 3) river routing. A sensitivity analysis (Fig. 3) revealed that the eight model parameters listed and described in Table 2, are most relevant for the calibration of runoff with this modelling system during the summer season. The semi-distributed parameters HSC (minimum soil water storage capacity) and HMX (maximum soil water storage capacity) define the amount of runoff generation. The runoff generation sub-module uses the hydrotope concept to consider the spatial variability of land use and soil related properties. The six remaining parameters $(\mathrm{C} 1, \mathrm{~S} 1, \mathrm{CC} 1, \mathrm{C} 2, \mathrm{~S} 2, \mathrm{CC} 2$; see Table 1) belong to the lumped runoff concentration module. They define the shape of the hydrograph. The catchment is partitioned into two types of contributing areas for the description of the runoff concentration processes, supplying mainly slow (uplands) and quick (hillslopes) runoff. Channel routing (with the Kalinin-Miljukov method) and snow melt can be simulated and optimized, but were not required in this study due to the particular case study areas and flood events that were chosen. 
Table 1. Summer flood events considered in this study.

\begin{tabular}{cccccc}
\hline Event & Begin & End & Duration & Peak flow Aue & Peak flow Schoenheide \\
\hline Jun 95 & $1995.06 .01,01: 00$ & $1995.06 .04,20: 00$ & $92 \mathrm{~h}$ & $32.5 \mathrm{~m}^{3} / \mathrm{s}$ & $13.7 \mathrm{~m}^{3} / \mathrm{s}$ \\
Sep 95 & $1995.08 .31,00: 00$ & $1995.09 .03,23: 00$ & $96 \mathrm{~h}$ & $144.1 \mathrm{~m}^{3} / \mathrm{s}$ & $84.1 \mathrm{~m}^{3} / \mathrm{s}$ \\
Jul 96 & $1996.07 .07,13: 00$ & $1996.07 .13,09: 00$ & $140 \mathrm{~h}$ & $64.1 \mathrm{~m}^{3} / \mathrm{s}$ & $31.6 \mathrm{~m}^{3} / \mathrm{s}$ \\
Sep 98 & $1998.09 .14,00: 00$ & $1998.09 .19,12: 00$ & $132 \mathrm{~h}$ & $61.9 \mathrm{~m}^{3} / \mathrm{s}$ & $41.2 \mathrm{~m}^{3} / \mathrm{s}$ \\
Nov 98 & $1998.11 .01,00: 00$ & $1998.11 .02,23: 00$ & $48 \mathrm{~h}$ & $72.7 \mathrm{~m}^{3} / \mathrm{s}$ & $31.6 \mathrm{~m}^{3} / \mathrm{s}$ \\
\hline
\end{tabular}

Table 2. Parameters of the ArcEGMO model.

\begin{tabular}{lllllccc}
\hline Parameter & Units & Type & Spatial units & Description & Initial value & Lower limit & Upper limit \\
\hline HSC & $\mathrm{mm}$ & Semi-distributed & Hydrotope & min. soil storage capacity & - & - & - \\
HMX & $\mathrm{mm}$ & Semi-distributed & Hydrotope & max. soil storage capacity & - & - & - \\
C1 & day & Lumped & Uplands & storage coefficient of SG & 150 & 30 & 270 \\
S1 & mm & Lumped & Uplands & storage capacity of SG & 120 & 25 & 215 \\
CC1 & day & Lumped & Uplands & storage coefficient of SGs & 8 & 1.6 & 1 \\
C2 & day & Lumped & Hillslopes & storage coefficient of SH & 5 & 16 \\
S2 & mm & Lumped & Hillslopes & storage capacity of SH & 20 & 4 & 35 \\
CC2 & day & Lumped & Hillslopes & storage coefficient of SHs & 1 & 0.2 & 1.8 \\
\hline
\end{tabular}

\section{A combined multi-objective optimization strategy}

\subsection{Definition of objective functions}

In semi-distributed models, some parameters with low spatial variability or low sensitivity are assigned to the entire catchment (lumped parameters). In this study, six of the eight parameters under consideration are of lumped type. As a consequence, if the parameter set changes, then the model performance may increase for some of the sub-basins, but decrease for others. Nevertheless, assigning these parameters to the smallest spatial units of the model as distributed parameters would contradict the aim of computational efficiency for real time forecasting. A compromise is the generation of nested sub-models (catchment zones) with individual lumped parameters. In order to find groups of sub-basins which could form such a zone, the relationship of parameterization among different sub-basins was considered by using the individual sub-basin as an objective function. The performance measure $E F F$, calculated from the transformed Nash Sutcliffe coefficient $N S^{*}$, is defined as

$E F F=\frac{1}{m} \sum_{i=1}^{m} N S_{i}^{*}$

with

$N S^{*}=1-N S=\sum_{j=1}^{n}\left(Q_{\mathrm{obs}, j}-Q_{\mathrm{sim}, j}\right)^{2} / \sum_{j=1}^{n}\left(Q_{\mathrm{obs}, j}-\bar{Q}_{\mathrm{obs}}\right)^{2}$, where $m$ is the number of simultaneously evaluated periods (flood events in this study), $n$ is the number of observations during a period, $j$ accounts for the time step and $Q$ is the discharge. The subscripts "sim" and "obs" indicate the simulated and observed values. The Nash-Sutcliffe coefficient NS in Eq. (3) is a measure for the overall agreement between observed and simulated time series (with $N S=1$ being the best). Here $N S^{*}$ is used in order to minimize the objective function values.

3.2 Methodology of the combined multi-objective optimization

According to different ways of interpreting the calibration process, the multi-objective optimization can be separated into a "stepped" and an "all at once" process (Fenicia et al., 2007). The "all at once" optimization process refers to the concept of Pareto-optimality (Gupta et al., 1998) and calibrates all model parameters simultaneously, considering a common set of objective functions. Such approaches result in Pareto-optimal solutions that represent the different trade-offs between parameters and objective functions. The "stepped" optimization assumes that each parameter can be assigned to a particular model process which influences the overall system response. Thus the parameter related to these objective functions can be calibrated in separate steps. The parameters of previous steps have to be fixed in the next calibration step. Consequently, a single solution that represents a balance between all objective functions should be obtained 
(a)

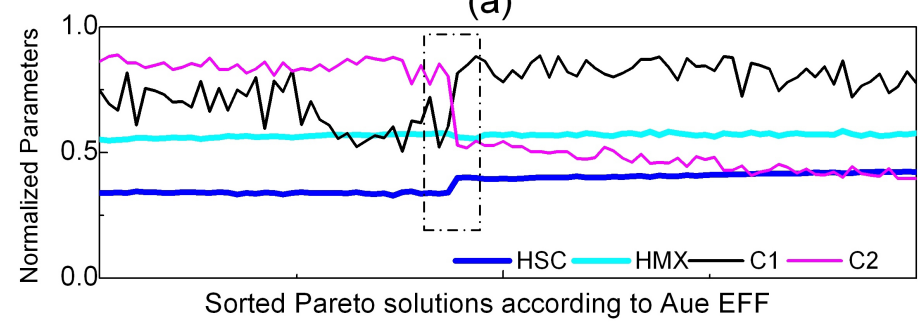

(c)

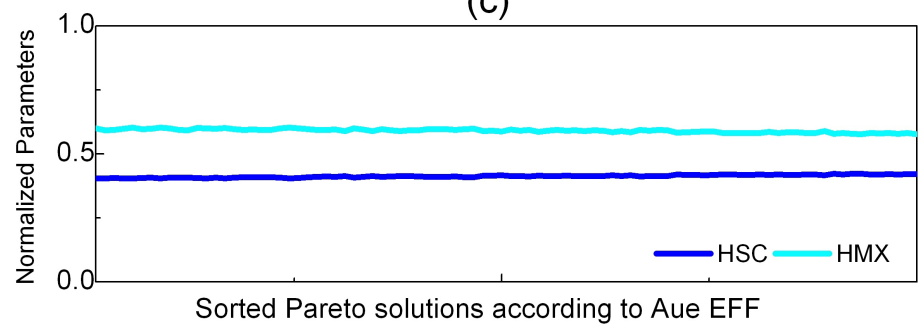

(e)

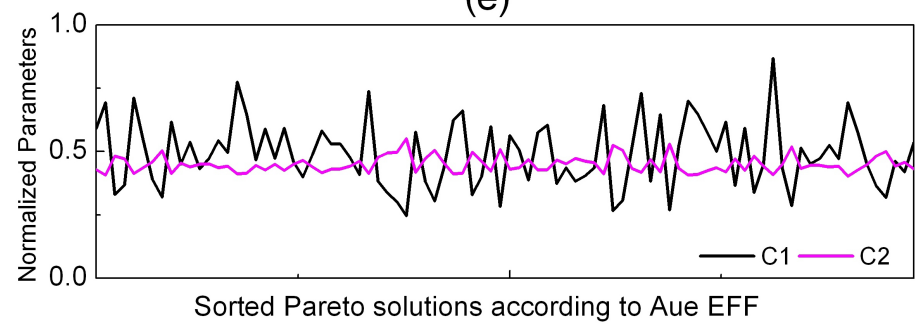

(b)

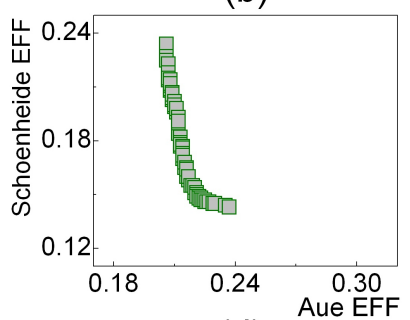

(d)

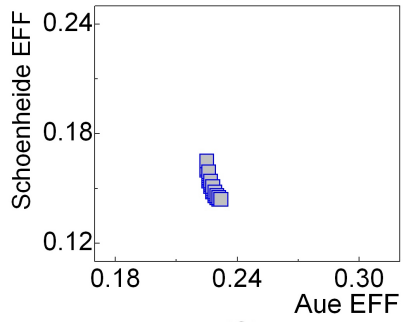

(f)

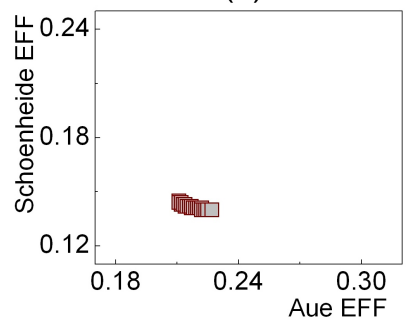

Fig. 4. "Stepped" initial optimization phase: (a) Parameter tendencies of step 1 (the dashed box indicates the region with a strong change in the parameter values), and (b) the corresponding Pareto-optimal front; (c) Parameter tendencies of step 2, and (d) the corresponding Pareto-optimal front; (e) Parameter tendencies of step 3, and (f) the corresponding Pareto-optimal front.

(Houge et al., 2003). Such an approach can provide a better performance than "all at once" calibration, but depends strongly on the parameter's identifiability and is of subjective character.

In this study, a method combining the benefits of a "stepped" and "all at once" optimization is suggested. This method is implemented in two phases: 1) the "stepped" initial optimization phase and 2) the "all at once" fine-tune optimization phase. In the first phase the optimization is investigated with different sets of parameters. Parameters with best identifiability are selected. In the second phase, the remaining parameters are optimized simultaneously. For this purposes the MOCOM (Multi-Objective COMplex evolution method, Yapo et al., 1998) was adopted.

\section{Case study}

\subsection{Initial optimization phase - stepped}

Step 1: The parameters HSC, HMX, C1 and C2 were optimized in this step, whereas the other parameters remained unchanged at their initial values. It should be noted that the runoff generation parameters HSC and HMX control the entire entry volume into the storage elements of the runoff concentration sub-model, whereas the baseflow parameters $\mathrm{C} 1$ and $\mathrm{C} 2$ are responsible for the amount of water that will be temporarily kept in the storage elements. These parameters were taken into account for the initial optimization phase, where 100 Pareto-optimal solutions were calculated. These 100 solutions allow for statistically significant conclusions and remain computationally inexpensive. The individual parameter tendencies, sorted according to the objective function value EFF at Aue gauge, are shown in Fig. 4a. Two strongly different parameter tendencies emerge from Fig. 4a. The runoff generation parameters are rather constant, whereas the baseflow parameters exhibit strong fluctuations. Furthermore, a sudden change, marked with a dashed box in Fig. 4a, has been observed. This phenomenon is termed specific parameter interaction, since the influence among the different parameters exists either solely in one or in a certain part of the entire set of solutions. We also note that the parameter HSC, previously found to be most sensitive (Fig. 2), is influenced through the variation of the less sensitive parameters $\mathrm{C} 1$ and $\mathrm{C} 2$. The Pareto-optimal front with the corresponding solutions can be seen in Fig. 4b. 

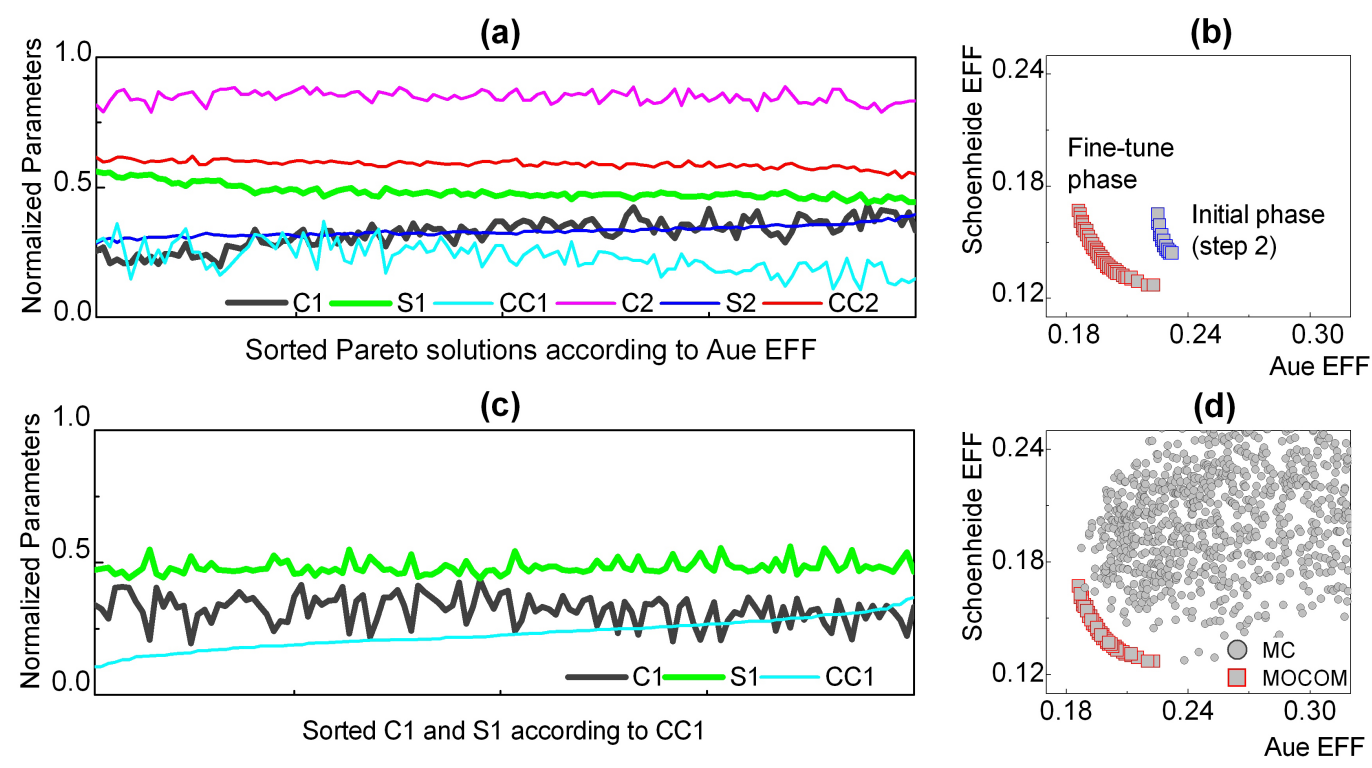

Fig. 5. "All at once" fine-tune optimization phase: (a) Parameter tendencies of fine-tune phase and (b) their corresponding Pareto-optimal front; (c) Sorted parameter tendencies to show the general parameter interaction; (d) Comparison of Pareto-optimal front from MOCOM, together with Monte-Carlo (MC) samples.

It is feasible to derive important information from the different parameter tendencies. HSC and HMX are typical catchment parameters which are parameterized by using soil and land use information. These parameters are therefore physically-based rather than conceptual. They are catchment-specific and independent from a particular flood event. Chappell et al. (2004) define such parameters as "properties of a catchment that are largely unchanging with time (e.g., soil-rock permeability, porosity), but may be spatially variable". By using ArcEGMO, spatial variability was considered using GIS-data in the pre-processing. On the other hand, the reservoir parameters of runoff concentration can be regarded as conceptual. These are influenced by both the catchment's characteristics and each flood event's specifications. For this reason, it is imperative to separate the runoff generation parameters from the reservoir parameters of runoff concentration during the optimization.

Step 2: In this step, HSC and HMX were optimized and the six remaining parameters of runoff concentration remained unchanged. The results of this step are shown in Figs. $4 c$ and $d$. The effect of local parameter interaction was avoided due to a reduction of the parameter members that were optimized. Both parameters show very little deviation from linear behavior. Therefore these parameters are identifiable for the sub-basins. Since the degree of freedom for the simulation is reduced, the extent of the Pareto-optimal front is also reduced. This will be addressed in the next step by using fixed values of HSC and HMX.

Step 3: The parameters $C 1$ and $C 2$ were optimized with fixed parameters HSC and HMX from step 2 and the other parameters remained unchanged with their initial values. The results of this step are shown in Figs. 4e and f. The comparison between the Pareto-optimal front of step 2 (Fig. 4d) and step 3 (Fig. 4f) shows significant improvement, in particular for the objective function of the Aue gauge. However, in Fig. 4e an opposing effect between different parameter tendencies of $\mathrm{C} 1$ and $\mathrm{C} 2$ can be observed. This is termed general parameter interaction that exists in all fields of the entire Pareto-optimal solutions. The consequence is large decision uncertainty. The parameters $\mathrm{C} 1$ and $\mathrm{C} 2$ therefore need to be estimated with the other parameters of runoff concentration in a subsequent fine-tune optimization phase.

\subsection{Fine-tune optimization phase - all at once}

In this phase, the six runoff concentration parameters are optimized with fixed HSC and HMX (Sect. 4.1, step 2) simultaneously. Figure 5a shows the individual parameter tendencies of 100 Pareto-optimal solutions. Figure 5b represents their corresponding Pareto-optimal front. The Paretooptimal front moved strongly to the axis origin when compared with the solution obtained during the initial optimization phase. This demonstrates an improved model performance that has been achieved through the combined optimization method. Figure 5a indicates that there is no significant change of the individual parameter tendencies. However, if only three directly related parameters $(\mathrm{C} 1, \mathrm{~S} 1$ and $\mathrm{CC} 1)$ are considered and subsequently sorted according to the value of CC1 (Fig. 5c), a similar opposing effect that has also been observed in Fig. 4e (i.e. general parameter interaction), in this case between $\mathrm{S} 1$ and $\mathrm{C} 1$, becomes evident. 
To confirm the validity of the Pareto front obtained with MOCOM, Monte-Carlo simulations (10000 parameter sets, uniform sampling) were employed additionally (Fig. 5d). The Monte-Carlo simulations did not yield values smaller than Aue EFF or Schoenheide EFF, i.e. the Pareto front of MOCOM, hence supporting the usefulness of the combined multi-objective optimization algorithm.

\section{Conclusions and outlook}

The analysis of parameter interaction shown here strongly supports the usefulness of an optimization strategy that reduces the structural uncertainties of conceptual rainfallrunoff models. Such a strategy was implemented in this study as a combined optimization scheme of a "stepped" and an "all at once" approach. For meso- to macroscale model applications further refinement of the model structure can be achieved by nesting regions. These nested regions are then treated as semi-distributed sub-models in the overall model setup. Currently the development of a concept for adaptive structuring of a nested watershed model is underway, whereby sub-basins, defined as objective functions, form the basis. It should be noted that such an approach may also significantly increase computational efficiency, which in turn plays an important role in probabilistic real time flood forecasting.

Edited by: K.-E. Lindenschmidt

Reviewed by: T. Weichel and H. Bormann

\section{References}

Becker, A., Kloecking, B., Lahmer, W., and Pfuetzner, B.: The hydrological modelling system Arc/EGMO, in: Mathematical models of large watershed hydrology, edited by: Singh, V. P. and Frevert, D. K, Water Resources Publ., Highlands Ranch, Colorado, USA, 321-384, 2002.

Chappell, N. A., Bidin, K., Sherlock, M. D., and Lancaster, J. W.: Parsimonious spatial representation of tropical soils within dynamic, rainfall-runoff models, in: Forests-Water-People in the Humid Tropics, Cambridge University Press, Cambridge, 85-98, 2004.

Duan, Q., Gupta, V. K., and Sorooshian, S.: Effective and efficient global optimization for conceptual rainfall-runoff models, Water Resour. Res., 28, 1015-1031, 1992.

Duan, Q., Gupta, V. K., and Sorooshian, S.: A shuffled complex evolution approach for effective and efficient global minimization, J. of Optimization Theory and Applications, 76(3), 501521, 1993.

Fenicia, F., Savenije, H. H. G., Matgen, P., and Pfister, L.: A comparison of alternative multi-objective calibration strategies for hydrological modeling, Water Resour. Res, 43, W03434, doi:10.1029/2006WR005098, 2007.

Gupta, H. V., Sorooshian, S., and Yapo, P. O.: Toward improved calibration of hydrologic models: Multiple and noncommensurable measures of information, Water Resour. Res, 34(4), 751764, 1998.

Hogue, T. S., Gupta, H. V., Sorooshian, S., and Tomkins, C. D.: A multi-step automatic calibration scheme for watershed models, Calibration of watershed models, Water Sci. Appl., 6, 165-174, 2003.

Vrugt, J. A., Bouten, W., Gupta, H. V., and Sorooshian, S.: A shuffled complex evolution Metropolis algorithm for optimization and uncertainty assessment of hydrological model parameters, Water Resour. Res, 39(8), 1201, doi: 10.1029/2002WR001642, 2003a.

Vrugt, J. A., Gupta, H. V., Bastidas, L. A., Bouten, W., Sorooshian, S.: Effective and efficient algorithm for multiobjective optimization of hydrologic models, Water Resour. Res., 39(8), 1214, doi:10.1029/2002WR001746, 2003b.

Yapo, P. O., Gupta, H. V., and Sorooshian, S.: Multi-objective global optimization for hydrologic models, J. Hydrol., 204, 8397, 1998 